\title{
Mineração de Dados em Logs de Auditoria como recurso no Processo de Ensino-Aprendizagem em Aulas Práticas de Banco de Dados
}

\author{
Késia Seles Lopes de Oliveira, Amanda Noronha da Silva, Jamilson Bispo dos \\ Santos, Leandro A. Silva
}
Faculdade de Computação e Informática - Universidade Presbiteriana Mackenzie São Paulo - Brasil

kesiaseles@yahoo.com.br, amandanoronha.s@hotmail.com, \{jamilson,prof.leandro.augusto\}@ mackenzie.br

\begin{abstract}
The use of the audit database is a common practice adopted by the Information Technology teams as a way to monitor the routine of database users, in order to verify failure, use and etc. In Computer Science courses, on the other hand, the audit configuration in issues that involve database can register the student information during class. This article presents as a proposal to use in log file data mining techniques (where the audit holds records) for the discovery of difficulties of students in practical discipline of database in order to be an important tool for the teacher in the teachinglearning process.
\end{abstract}

Resumo. O uso da auditoria em Banco de Dados é uma prática comum adotada pelas equipes de Tecnologia da Informação como forma de monitorar a rotina dos usuários de banco de dados, afim de verificar falha, formas de uso e etc. Em cursos de Computação, por outro lado, a configuração da auditoria em disciplinas que envolvem banco de dados pode registrar informações de uso dos aluno durante as aulas. Este artigo apresenta como proposta o uso de técnicas de mineração de dados em arquivos de log (onde a auditoria armazena os registros) para a descoberta de dificuldades dos alunos em disciplina prática de Banco de Dados com o objetivo de ser um importante instrumento para o professor no processo de ensino-aprendizado.

\section{Introdução}

Com o avanço da tecnologia da informação (TI) facilitou-se o armazenamento eletrônico de informações, aumentando assim o volume de dados de tal maneira que se torna impossível alguma analise que assegure sobre sua confiabilidade (Imoniana, 2011). Dessa maneira, emerge a importância da auditoria, que consiste em um processo de coletar, agrupar e avaliar evidências para determinar se um sistema informatizado protege e mantém a integridade dos dados e se os propósitos da empresa foram 
aplicados de forma eficaz. A auditoria, em geral, é uma análise cuidadosa de alguma atividade que foi desenvolvida em uma empresa. Ela tem como objetivo verificar se o que está sendo analisado está de acordo com o que foi determinado previamente, ou se está seguindo as regras que foram pré-definidas em diferentes segmentos da TI (Velthuis e Navarro, 2001). Por exemplo, no caso dos Sistemas de Gerenciamento de Banco de Dados (SGBDs) a implementação da auditoria é um elemento sensível às empresas por considerar que os dados armazenados são matéria prima fundamental para a geração de relatórios ou indicadores que os gestores se baseiam em tomada de decisões (Gil, 2000).

Quando uma empresa configura seu SGBD com o módulo de auditoria, todas as transações realizadas nos sistemas automatizados são registradas em arquivos, que podem ser armazenados em diferentes formatos, gerados automaticamente para que os auditores possam analisar todas as operações que os usuários estão realizando e, de acordo com cada sistema, verificar se as operações são válidas ou se existe alguma particularidade como, por exemplo, alertas e erros (GRÉGIO, 2007). Esses arquivos são chamados de $\log s$ e sua análise não é simples, pois, dependendo da auditoria que se deseja fazer, é necessária a manipulação de todas as operações existentes nos logs ou então a limpeza do que não é necessário, sendo uma tarefa muito árdua (Silva, Boscarioli e Peres, 2003).

Considerando os aspectos supracitados como a importância de utilizar a auditoria de SGBD para analisar as transações realizadas e armazenadas na forma de log, este trabalha aborda a análise desse arquivo de dados oriundos de um Banco de Dados utilizado em aulas de laboratório de cursos de Computação. A ideia principal do trabalho é verificar se os alunos estão realizando de maneira correta os conceitos que estão sendo apresentados pelos professores. Como o volume de dados gerado é muito grande, as analises serão feitas com o uso de técnicas de mineração de dados (MD). Portanto, o objetivo do trabalho é utilizar agrupamento de dados, uma tarefa de MD, para analisar o arquivo de $\log$ gerado em aulas laboratoriais de cursos de Banco de Dados. Os resultados deste trabalho podem gerar resultados quantitativos e também qualitativos para avaliar o aprendizado dos alunos nesse tipo de disciplina.

A metodologia deste trabalho consiste na configuração do módulo de auditória do SGBD Oracle versão $11 \mathrm{~g}$, definição de um período de coleta para restringir o assunto que está sendo abordado em aula e uso do software Rapid Miner para a execução do algoritmo de agrupamento de dados $k$-médias (ou $k$-means) e análise dos resultados.

Além da introdução que apresenta a motivação e objetivo deste trabalho, o artigo está estruturado em outras três seções. Na 2 apresenta-se o conceito de Mineração de Dados, o uso desse conceito em análise de texto e uma rápida apresentação sobre agrupamento de dados. Na seção 3 apresenta-se a metodologia dos experimentos e os resultados. Por fim, conclui-se sobre o artigo e os resultados obtidos.

\section{Mineração de Dados}

Mineração de Dados (MD) é uma área de pesquisa multidisciplinar, envolvendo basicamente Banco de Dados, Estatística e Aprendizado de Máquina. A MD é parte principal de um processo que tem como entrada uma Base de Dados e como saída um Conhecimento (Fayyad et al., 1996). Ela é divida em tarefas como predição, 
clusterização e associação de dados que devem ser escolhidas de acordo com analises exploratórias inicialmente feitas sobre os dados (Han et al., 2006; Tan et al., 2009; Faceli et al., 2011; Silva, 2015). A MD tem sido amplamente utilizada em diferentes áreas, inclusive na educação. Quando se usa para analise dados envolvidos com a educação, como neste trabalho, constuma-se definir a área de pesquisa como Mineração de Dados Educacionais ou MDE (Silva \& Silva, 2014; Romero \& Ventura,2010).

\subsection{Análise de Agrupamento}

A descoberta de grupos ou clustering trata-se de um modelo que procura encontrar exemplares ou padrões com atributos semelhantes na base de dados. Portanto, a segmentação da base em grupos é feita a partir de medidas de similaridade. Em problemas que envolvem agrupamento não se encontra disponível o atributo especial classe ou rótulo. Por esta razão, dizemos que o aprendizado realizado pelos algoritmos de agrupamento é não-supervisionado (Jain \& Dubes, 1988; Han et al., 2006; Tan et al., 2009; Jain, 2010; Faceli et al., 2011; Witten et al., 2001; Silva, 2015).

O aprendizado não-supervisionado é de grande desafio, pois não se tem o objetivo que se deseja alcançar, o que significa não conhecer o número de grupos da base de dados. $\mathrm{E}$ ainda, os exemplares estão distribuídos em um espaço de dimensão elevada com diferentes formatos e separação. Estes aspectos ilustram a grande dificuldade para lidar em problemas de agrupamento de dados.

Em um contexto geral, o que o agrupamento faz é a descoberta de perfil. Mais especificamente em educação, a descoberta poderia ser útil para descobrir estilos de aprendizado dos alunos, padrões de erros, disciplinas de interesses comuns e muitas outras aplicações.

Os algoritmos de agrupamento também podem ser divididos em Aprendizado de Máquina e Inteligência Computacional. Algoritmos típicos de Aprendizado de Máquina são o Agrupamento Hierárquico, k-Médias (k-Means), Agrupamento Espacial baseado em Densidade ou DBScan (Density Based Spatial Clustering of Applications with Noise). Por outro lado, como abordagem baseada em Redes Neurais têm o Mapa AutoOrganizável ou SOM (Self-Organizing Map) (Jain \& Dubes, 1988; Han et al., 2006; Tan et al., 2009; Haykin, 2009; Jain, 2010; Faceli et al., 2011; Witten et al., 2001). A seguir será apresentado em detalhes o K-Médias, algoritmo a ser utilizado neste estudo.

\subsubsection{Algoritmo $k$-Médias}

O $k$-Médias ou, em inglês, $k$-Means é o principal algoritmo de agrupamento particional (Partitional Clustering) (Silva, 2015). O objetivo é encontrar particionamentos nos exemplares da base de dados dentro de $k$ grupos disjuntos. No entanto, o número de grupos representado por $k$ deve ser dado como um parâmetro de entrada do algoritmo, como também a medida de distância - geralmente a Euclidiana. Com os parâmetros de entrada definidos é feita uma escolha aleatória de $k$ distintos valores para centroides de grupos. E a partir destes centroides, o algoritmo iterativo gera as partições na base, fornecendo como resultado final a base de dados segmentada. O Algoritmo 1 descreve todos os passos deste processo de agrupamento de dados.

O algoritmo $k$-Médias explicado nesta seção espera que os exemplares parem de fazer troca de centroides em alguma iteração, o que pode não acontecer. Por esta razão, podese considerar uma função objetivo para que seja minimizada (ou maximizada) até um 
V Congresso Brasileiro de Informática na Educação (CBIE 2016)

Anais dos Workshops do V Congresso Brasileiro de Informática na Educação (CBIE 2016)

valor aceitável como critério de parada do algoritmo ou que ao menos se defina um número máximo de iterações para que o algoritmo se estabilize (Tan et al., 2011).

Algoritmo 1 - Algoritmo $k$-Médias

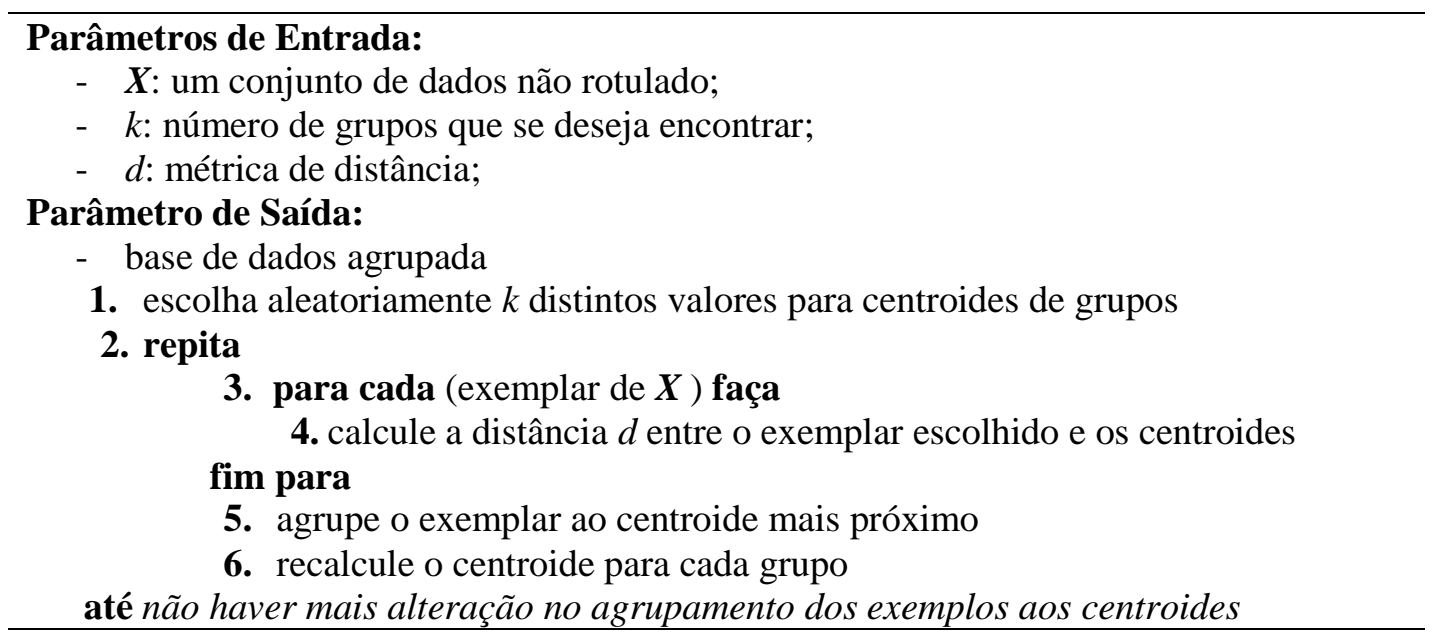

Em problemas de agrupamento o desempenho do algoritmo pode ser medido através do uso de índice de validação de agrupamento (em inglês, cluster validity indices) e os métodos se dividem em interno, relativo e externo (Jain \& Dubes, 1988; Jain, Murty e Flynn, 1999). Os índices internos baseiam-se na estrutura gerada pelo algoritmo de agrupamento. Portanto, a grande maioria dos quantificadores mensuram a coesão e o isolamento dos grupos. Exemplos de índices internos são Coeficiente de Silhoueta, Índice de Dunn, Índice de Davies-Bouldin entre outros.

\subsection{Mineração de Texto}

Como as instruções armazenadas no $\log$ estão em formato de texto, para aplicação do algoritmo de agrupamento é necessário estruturar os dados por meio de um modelo de representação, que transforma os termos de cada instrução em um valor de relevância (peso). O resultado disso é chamado de corpus, e a partir desse, é que o algoritmo de agrupamento é executado. Esse processo ocorre da seguinte maneira (Göker, Davies, 2009):

i. Tokenization: a partir do caractere espaço, os comandos das instruções são separadas em tokens. Os caracteres especiais como vírgulas (","), pontos de exclamação ("!") e de interrogação ("?"), entre outros são removidos, assim como números.

ii. Stopwords: palavras como preposições, pronomes, artigos, advérbios que são comuns em diferentes contextos são removidas do processo;

iii. Stemming: as palavras resultantes das etapas anteriores passam por uma normalização ortográfica para que sejam reduzidas ao radical. Este processo é 
importante, pois permite que palavras com o mesmo radical sejam consideradas como semelhantes.

Ao final deste processo de transformar um texto em um corpus, as palavras são armazenadas em seus respectivos vocabulários.

A representação dos documentos é feita usando um modelo chamado espaço vetorial (Göker, Davies, 2009). O modelo espaço vetorial busca representar documento em forma de vetor, composto por termos (palavras) representados por um peso ponderado. Uma das formas de se fazer a ponderação é combinando a frequência que um termo aparece em um documento, que aqui neste trabalho será uma instrução SQL, ( $f$ do inglês, term frequency) com a frequência invertida do documento (idf do inglês, inverse document frequency). A combinação de $t f \mathrm{x}$ idf define a importância (peso) de um termo dentro do documento (Göker, Davies, 2009).

Assim, depois de calculado o peso de cada termo do documento, tem-se como resultado uma base de dados numérica formada por vetores de documentos e, assim, consequentemente, aplica-se o algoritmo de agrupamento de dados.

\section{Metodologia e Resultados Experimentais}

Para a realização dos experimentos, utilizou-se como base de dados os logs do Oracle $11 \mathrm{~g}$ referentes a aulas práticas de banco de dados de cursos de uma faculdade de Computação. Os experimentos de mineração de dados e análise foram feitos utilizando a ferramenta Rapid Miner.

Como os conceitos de um curso são amplos, decidiu-se subdividir a base em assuntos específicos, partindo do princípio que a cada semana os professores aplicam conceitos diferentes com exercícios que são realizados no mesmo período, por exemplo, em uma determinada semana o assunto abordado em aula se refere às funções agregadas de SQL (MAX, MIN, AVG, COUNT, SUM) e, consequentemente, os exercícios exigem o uso das mesmas. Assim, os logs daquele período podem ser analisados para verificar a forma como os alunos estão utilizando os conceitos passados em sala.

Para a configuração da auditoria é necessário que o usuário tenha privilégios de administrador do banco de dados. O processo de configuração se dá a partir dos seguintes passos: Habilitação do parâmetro audittrail que contém a informação base para realização deste experimento; Configuração do audit_trail usada para definir que tipo de instrução será armazenada na tabela de $\log s$, por exemplo, neste trabalho foi permitido salvar instruções do tipo SELECT, INSERT, DELETE, UPDATE e EXECUTE; Por fim, definição do arquivo de dados na tablespace que consiste em uma tabela auxiliar criada para não comprometer o desempenho do banco de dados.

O tablespace armazena desde informações simples até as mais complexas, e isto a torna excessivamente grande, ou seja, com grande quantidade de informações arquivadas de forma desestruturada. Isto acaba afetando o processamento das informações e levando um tempo acima do esperado para obter o resultado dos comandos executados pelos alunos em analise. Por este motivo foi criado um índice no campo USERID na tabela de auditoria (sys.aud\$) e, após isto, as consultas passaram a ter um tempo de execução menor.

O campo OBJ_NAME da tablespace diferencia o perfil do usuário que executou instruções no sistema, usuários, ou seja, pode-se separar o perfil de administrador, 
V Congresso Brasileiro de Informática na Educação (CBIE 2016)

Anais dos Workshops do V Congresso Brasileiro de Informática na Educação (CBIE 2016)

usuários que são professores e usuários que são alunos. Foi utilizado a seguinte query para extração dos dados:

\section{SELECT SQL_TEXT FROM SYS.AUD\$ WHERE OBJ_NAME = 'ALUNO'}

A tabela tablespace possui informações do usuário que realizou a consulta, data e hora, ações executadas, objetos selecionados, códigos das seções entre outras, que não serão utilizadas nesta pesquisa, mas que podem ser incorporadas em trabalhos futuros. Aqui foi escolhido apenas utilizar o SQL_TEXT que contém todas as instruções realizadas.

Os dados resultantes ficaram em formato de duas colunas, uma com um identificador de instrução e outra com a instrução realizada pelos alunos. Os resultados foram salvos em um arquivo no formato .csv. O Oracle oferece opção de escolha de formato na extração dos resultados. Após a coleta dos dados, o processo ilustrado na Figura 1 foi elaborado no Rapid Miner para a análise dos dados. Nesse processo, o passo inicial (1) consiste na leitura do arquivo com os dados do $\log$. Na fase seguinte (2), inicia-se a fase de mineração de texto (Seção 2.2). Nessa fase, todas as palavras presentes nas instruções foram colocadas em letra minúscula, espaços adicionais e pontuações foram removidas.

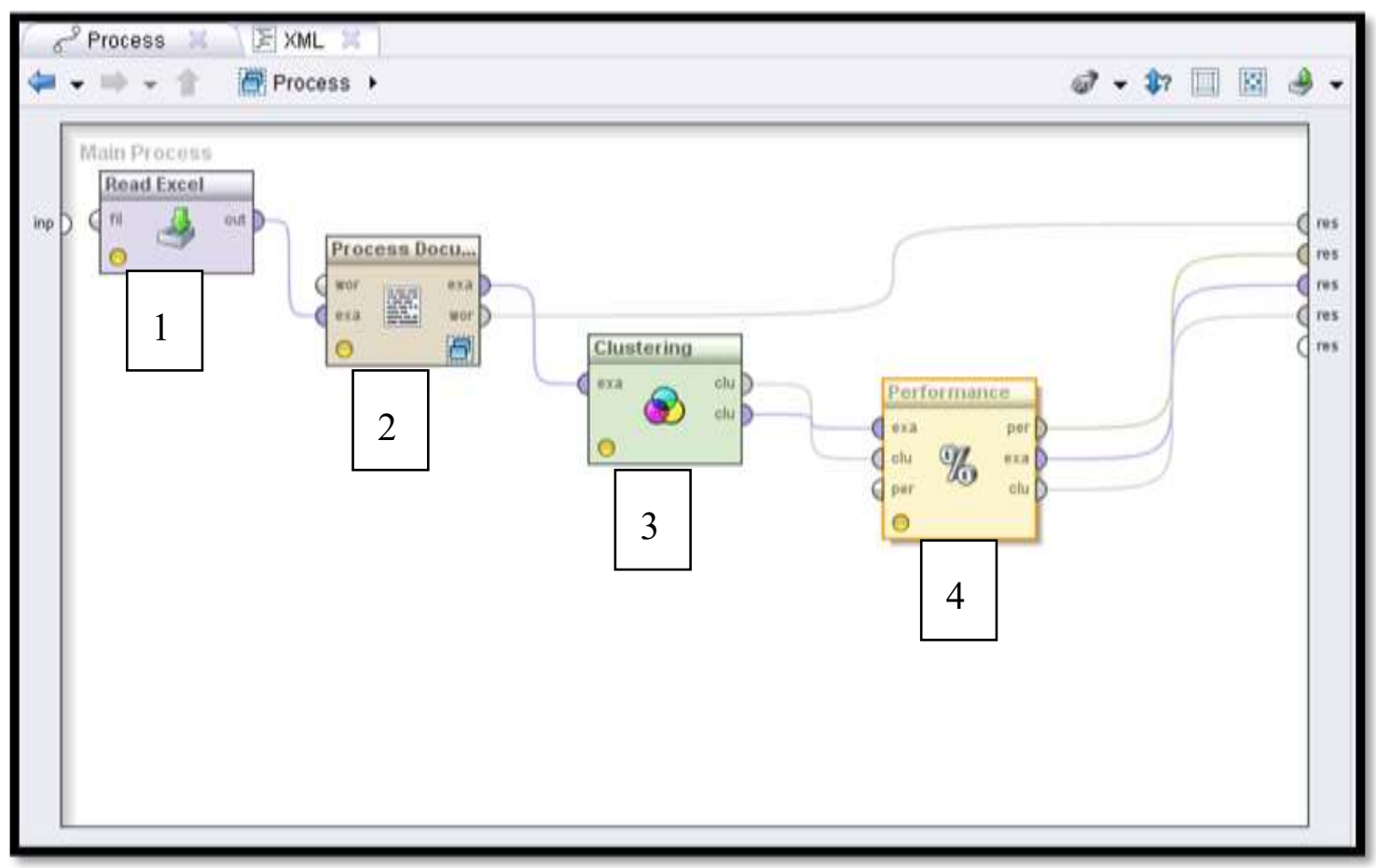

Figura 1: Processo para analise dos dados usando a tarefa de agrupamento (clustering) do Rapid Miner.

No passo 3 do processo ilustrado na Figura 1 executa-se o agrupamento, considerando nas analises dois grupos $(\mathrm{k}=2)$, ou seja, pensando-se em encontrar dois tipos de situações: instruções corretas e incorretas. $\mathrm{O}$ primeiro experimento realizado considerou os comandos SELECT simples, que é caracterizado pela consulta simples em uma tabela sem utilização de cláusulas. Na

Figura 2a está um primeiro resultado encontrado com a quantidade de instruções em 
V Congresso Brasileiro de Informática na Educação (CBIE 2016)

Anais dos Workshops do V Congresso Brasileiro de Informática na Educação (CBIE 2016)

cada grupo descoberto pelo algoritmo $k$-Médias. Em complemento, na

Figura 2b, está o resultado da contagem de cada termo da instrução. Em analise conjunta aos dois resultados, nota-se que o grupo identificado como Cluster_0 provavelmente representa os casos de instruções incorretas, pois veja que na

Figura 2b, aparecem comandos como FROMDB, FROMDBD,FROMMIBD e INTO, onde na verdade espera-se pela instrução correta que é o FROM. Portanto, este tipo de descoberta de conhecimento da análise de mineração de dados pode ser um indicador para o professor de qual conceito deve ser melhor enfatizado na exposição do assunto em aula ou mesmo elaborar atividades que exercitem o assunto.

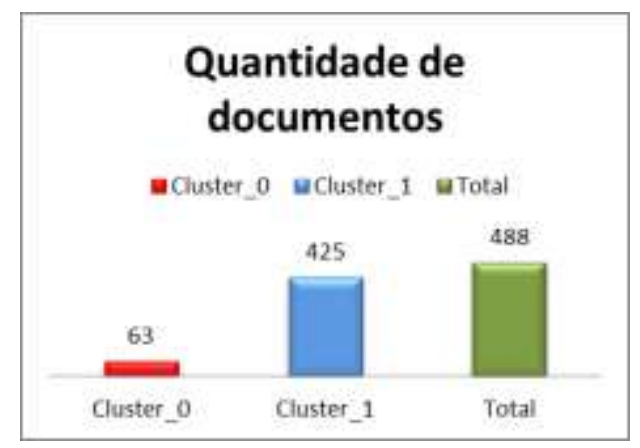

a) Número de instruções em cada agrupamento (cluster)

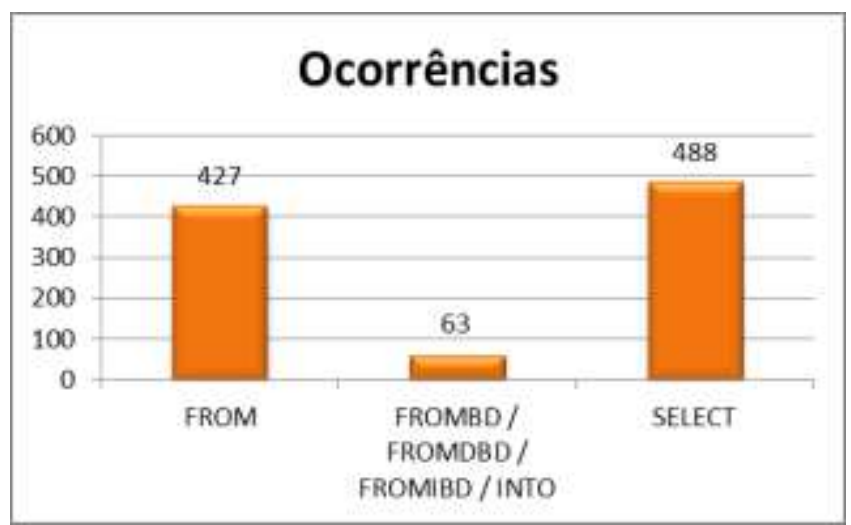

b) Termos presentes no conjunto de dados e quantidade de ocorrências

Figura 2: Resultados obtidos na analise da instrução SELECT

Após a análise de SELECT simples, foi realizado o processamento com SELECTs contendo a cláusula WHERE e outros comandos como BETWEEN e ORDER BY. Para realizar a análise, foi efetuada a mesma metodologia adotada no caso apresentado anteriormente na

Figura 2, porém com um número reduzido de alunos. Os resultados da analise estão apresentados na

Figura 3 e agora, nota-se que não há evidencias claras como no caso anterior, onde se pôde identificar o grupo de situações corretas e incorretas. Ou seja, as 17 instruções mapeadas no Cluster_0 não são evidentes de quais ocorrências levam a que tipo de situação, mesmo em análise conjunta à

Figura 3b. No entanto, analisando o resultado dessa última figura, nota-se que todas as instruções fazem corretamente a construção SELECT, WHERE e FROM (foram analisadas instruções de 64 alunos), ou seja, não tem os mesmos erros da situação da

Figura 2b. O uso do AND e do OR aparece na

Figura $3 \mathrm{~b}$ com a mesma quantidade, o que indica para esse ensaio que se foi trabalhado o uso conjunto dos dois operadores, o que pode ser útil em uma análise do professor, pois caso um ou outro estivesse em menor quantidade poderia significar uma 
V Congresso Brasileiro de Informática na Educação (CBIE 2016)

Anais dos Workshops do V Congresso Brasileiro de Informática na Educação (CBIE 2016)

compreensão equivocada do enunciado. Os comandos ORDER, BY e ASC, também aparecem com a mesma quantidade de ocorrência (treze), o que aparentemente aparenta ser um caso correto, uma vez que tais comandos devem ser executados conjuntamente. Uma última observação neste experimento é a quantidade de termos do tipo LIKE. Como três é uma quantidade relativamente pequena em relação ao número de instruções analisadas, isto pode representar que um aluno utilizou o comando sem haver a real necessidade, por exemplo:

\section{SELECT NOME FROM ALUNO WHERE (IDADE >=18 AND IDADE <=20) OR (BAIRRO LIKE '\%LAPA\%')}

\section{SELECT NOME_ALUNO FROM ALUNO WHERE (IDADE BETWEEN 18 AND 20) OR BAIRRO = 'LAPA' ORDER BY NOME_ALUNO ASC}

As duas instruções funcionam, porém os resultados aqui apresentados pode ajudar o professor a identificar se o aluno está utilizando apenas os conceitos que ele já conhece ou se está praticando os novos conceitos passados na aula referente ao período em que a base foi extraída.

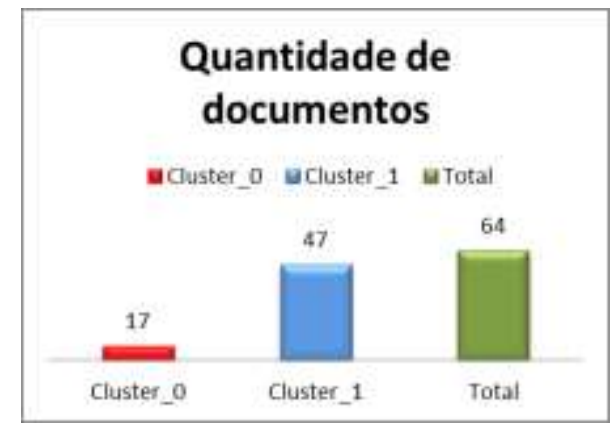

a) Número de instruções em cada agrupamento (cluster)

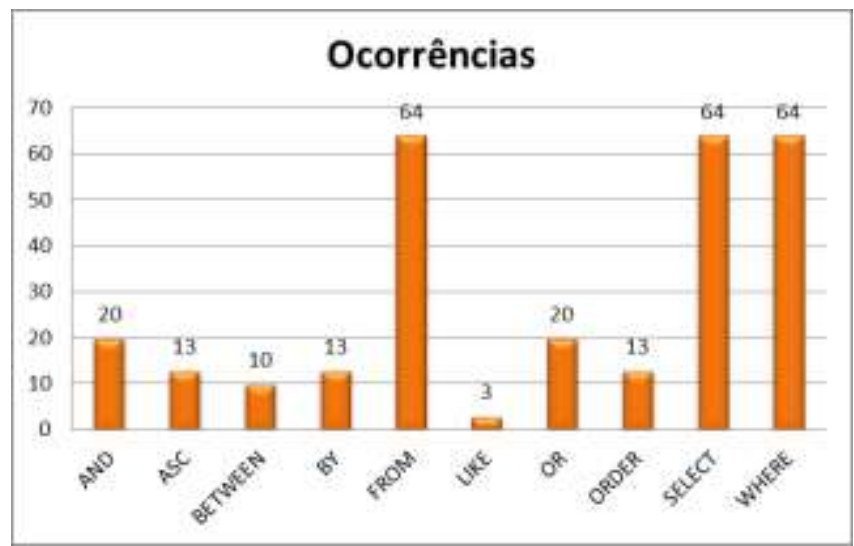

b) Termos presentes no conjunto de dados e quantidade de ocorrências

Figura 3: Resultados obtidos na analise da clausula SELECT e outros comandos

\section{Conclusão}

De forma geral, a mineração de dados auxilia na interpretação do professor sobre o comportamento dos alunos em sala de aula e desenvolvimento do aprendizado, porém, o processo deve ser acompanhado pelo professor que tem o conhecimento do tipo de atividade que foi proposta para o laboratório e, reconhecendo as evidencias usar os resultados de analise como diagnostico do conceito abordado.

Uma desvantagem identificada ao analisar os dados agrupados é o comportamento da ferramenta e do algoritmo em relação ao erro referente à ordem das instruções realizadas em uma query, como por exemplo, a utilização do GROUP BY e do HAVING. Com as análises realizadas durante o trabalho, pode-se perceber que a 
indiferença da ordem das duas instruções presentes nas instruções, sendo que o correto é primeiramente o uso do GROUP BY e depois o uso do HAVING. Ao realizar a mineração seria agrupado todos os documentos referentes a essas instruções em um mesmo grupo, pois apresentam termos semelhantes, porém não são iguais, pois a query que foi trocada a ordem está incorreta. Como sugestão para trabalhos futuros seria interessante a continuidade do estudo feito para este trabalho, porém com o intuito de encontrar alguma ferramenta ou algoritmo que consiga identificar outros padrões de erro, como no caso da ordem de instruções, que é essencial para construir as instruções com group by e having corretamente, mas que é um erro não mitigado pela ferramenta e algoritmo apresentado.

\section{Referencias Bibliográficas}

Faceli, K., Lorena, A. C., Gama, J., \& Carvalho, A. C. P. L. F. (2011). Inteligência Artificial: Uma Abordagem de Aprendizagem de Máquina,. LCT.

Fayyad, U.; Piatetski-Shapiro, G.; Smyth, P (1996). From Data Mining to Knowledge Discovery: An Overview. In Advances in Knowledge Discovery and Data Mining. California. p. 1-34.

Gil, A.L. (2000), Auditoria de Computadores, Ed. Atlas, São Paulo, $5^{\text {a }}$. Ed.

Göker, A., \& Davies, J. (2009). Information retrieval: searching in the 21st century. Chichester U.K.: John Wiley \& Sons, Inc.p.6-7.

Grecio, A. R. A. (2008), Aplicação de Técnicas de Data Mining para a Análise de Logs de Tráfego TCP/IP, Dissertação apresentada ao Instituto Nacional de Pesquisas Espaciais INPE para obtenção da pós-graduação em Computação Aplicada. São José dos Campos.

Han, J. \& Kamber; M. (2006): Data Mining Concepts and Tecniques. Editora Elsevier. Morgan Kaufmann, $3^{\text {rd }}$ edition.

Imoniana, J.O. (2011). Auditoria de Sistemas de Informação. São Paulo, Editora Atlas, $1^{\mathrm{a}}$. ed.

Jain, A. K., \& Dubes, R. C. (1988). Algorithms for clustering data. New Jersey: Prentice-Hall.

Jain, A.K.; Murty, M.N. \& Flynn, P.J. (1999). Data clustering: a review. ACM Comput Surv, New York, vol. 31, No 3, 264-323.

Romero, C., \& Ventura, S. (2010). Educational data mining: a review of the state of the art. Systems, Man, and Cybernetics, Part C: Applications and Reviews, IEEE Transactions on, 40(6), 601-618.

Silva, L. A. Mineração de dados: uma abordagem introdutória e ilustrada. Editora Mackenzie (Coleção conexão inicial, v. 11), 1. ed., São Paulo, 2015.

Silva, L. A., \& Silva, L. (2014). Fundamentos de Mineração de Dados Educacionais. In Anais dos Workshops do Congresso Brasileiro de Informática na Educação (Vol. 3, No. 1).

Silva, M.P., Boscarioli, C., Peres, S. M. (2003), Análise de Logs da Web por Meio de Técnicas de Data Mining. I Congresso de Tecnologia para Gestão de Dados e Metadados do Cone Sul (CONGED), Ponta Grossa..

Tan, P.-N., Steinbach, M., \& Kumar, V. (2009). Introdução ao datamining: mineração de dados. Rio de Janeiro: Ciência Moderna.

Velthuis, M. G.P. \& Navarro, .E.D.P (2001), Auditoría Informática: Un enfoque prático, Pitágoras, Alfaomega Grupo Editor, 2 ed.

Witten, I. H., Frank, E., \& Mark, A. (2011). Hall (2011)." Data Mining: Practical machine learning tools and techniques. 